\title{
Improved performance of perylenediimide-based lasers
}

\author{
Manuel G. Ramirez, ${ }^{a}$ Marta Morales-Vidal, ${ }^{a}$ Víctor Navarro-Fuster, ${ }^{a}$ Pedro G. Boj, ${ }^{b}$ José A. Quintana, ${ }^{b}$ José \\ M. Villalvilla, ${ }^{a}$ Aritz Retolaza, ${ }^{c}$ Santos Merino ${ }^{c}$ and María A. Díaz-García ${ }^{* a}$
}

Received (in $X X X, X X X)$ Xth $X X X X X X X X X 20 X X$, Accepted Xth $X X X X X X X X X 20 X X$

DOI: 10.1039/b000000x

\begin{abstract}
The aim of this work was to improve the laser performance, in terms of threshold and operational lifetime, of lasers based on polymer films doped with perylenediimide (PDI) derivatives as active media. For such purpose, we first investigated the amplified spontaneous emission (ASE) properties of perylene orange (PDI-O), when doped into polystyrene (PS) films. Lower ASE thresholds and larger photostabilities than those of similar films containing another PDI derivative (PDI-C6), recently reported in the literature, have been 10 measured. Results have been interpreted in terms of the photoluminescence efficiency of the films, which depends on the type of molecular arrangement, inferred with the help of nuclear magnetic resonance experiments. We also show that PS films have a better ASE performance, i.e. lower thresholds and larger photostabilities, than those based on poly(methyl methacrylate), which was recently highlighted as one of the best matrixes for PDI-O. Finally, a 1D second-order distributed feedaback laser using PS doped with PDI-O, was fabricated and characterized. This device has shown a threshold significantly lower (by around one order of magnitude) than that of 15 a similar laser based on PDI-C6-doped PS.
\end{abstract}

\section{Introduction}

In the last years extensive research has been devoted to the search of materials with optimal properties to be applied in the 20 development of organic solid-state lasers (OSLs). ${ }^{1-4}$ Organic materials offer various advantages, such as easy processability in the form of thin films, chemical versatility, wavelength tuneability and low cost. The interest in OSLs increased with the discovery of stimulated emission in optically-pumped 25 semiconducting polymer films, ${ }^{5,6}$ since they opened the possibility of using electrical excitation. The goal of obtaining laser diodes was initially the main motivation to decrease the pump intensity needed, i.e. the threshold, to obtain laser emission from OSLs, so many works focused on improving the active 30 materials and the resonators. Lasing by electrical injection has not been demonstrated yet. However, thanks to all these efforts, laser thresholds have been decreased so much that today it is possible to pump with cheap inorganic diode lasers ${ }^{3}$ and even with light emitting diodes. ${ }^{7}$ Therefore, these low-cost and compact 35 optically-pumped lasers are by themselves useful for applications. ${ }^{1,2}$

Distributed feedback (DFB) resonators have been widely used to buid OSLs. ${ }^{2-4}$ In DFB lasers, feedback is achieved by the 40 incorporation of periodic nanostructures (obtained by modulating either the refractive index or the gain) that Bragg-scatter the light, thus avoiding the need of good-quality end facets. In addition, these resonators can be easily integrated into planar organic waveguides, which is a clear advantage from the fabrication point 45 of view, as compared to other types of laser cavities. DFBs have shown great potential for the development of applications in the fields of telecommunications, ${ }^{4}$ biosensing and chemical sensing. ${ }^{3,8,9}$ From a material's point of view, the simplest way to assess the laser properties of a given material for waveguide50 based laser devices, such as DFBs, consists in studying its amplified spontaneous emission (ASE) properties when deposited as a thin film in a waveguide configuration. ${ }^{2,10}$ Typically, the active film is photopumped with a pulsed optical source, aiming to identify a collapse of the width of its photoluminescence 55 spectrum and a large enhancement of the output intensity above the threshold. A combined characterization of the ASE and the DFB properties allows to optimize separately the active material and the laser cavity.

${ }_{60}$ A wide variety of materials have been used to fabricate the active layers of organic DFBs. ${ }^{1-4}$ Among them, our research group has focused in the last years in polystyrene (PS) films doped with perylenediimide derivatives (PDIs). ${ }^{11-17}$ In some of the initial studies, the aim was to analyze the effect of modifying the ${ }_{65}$ chemical structure of the PDIs in their spectral, electrochemical and ASE properties when diluted in liquid solutions as well as in PS films at various concentrations. ${ }^{11-13}$ The results obtained through those works allowed to reach important conclusions from the molecular point of view. The best ASE performance was 70 obtained with PDIs symmetrically substituted at the imide nitrogen positions. The PDI derivative N,N'-di(10nonadecyl)perylene-3,4:9,10-tetracarboxylic diimide (denoted as PDI-N in ref. 12) showed photostable ASE at low thresholds, at doping concentrations into PS of only $0.5-1 \mathrm{wt} . \%$. Other types of 75 modifications, such as substitutions at the bay positions in the PDI core or modifications in the dicarboximide group lead to a much worse performance. Therefore, in subsequent studies, PDI derivatives substituted in the $\mathrm{N}$ positions were used. In particular, high quality second-order one-dimensional (1D) DFB lasers, with 
gratings engraved both on the substrate ${ }^{14,15}$ and on the active film, ${ }^{16}$ have been fabricated by using PS doped with the PDI derivative N,N'-di-(1-hexylheptyl) perylene-3,4:9,10tetracarboxylic diimide (PDI-C6, see Fig. 1(a)). The chemical 5 structures of PDI-C6 and PDI-N are very similar, since they are both symmetrically substituted at the $\mathrm{N}$ atoms with alkyl chains. They only differ in the length of these chains. The ASE properties of PS films doped with $0.5 \mathrm{wt} . \%$ of PDI-C6 are similar to those of films containing PDI-N. ${ }^{17}$ DFB lasers based on PDI-C6 have 10 shown a very good laser performance: emission wavelength between 554 to $583 \mathrm{~nm}$ (depending on film thickness and grating depth), thresholds of $1 \mu \mathrm{J} /$ pulse, single-mode emission with linewidths below $0.2 \mathrm{~nm}$ and photostability half-lives of $\sim 10^{5}$ pump pulses under excitation in ambient conditions.

The laser properties of PDI-doped solid matrixes have also been investigated by other authors, ${ }^{18-27}$ motivated mainly by the high thermal and photostability of PDIs. In most studies, the commercially available derivatives known as perylene orange

20 (N,N'-di(2,6-diisopropylphenyl)-3,4:9,10-tetracarboxylic

diimide, PDI-O, see Fig. 1(b)) and perylene red were used. PDI-O belongs to the class of PDI derivatives symmetrically substituted at the imide nitrogen positions. Recently, the role of the matrix in optimizing the ASE properties of PDI-O has been investigated. ${ }^{27}$ 25 In that work, poly(methyl methacrylate) (PMMA) was highlighted as one of the best matrixes, from the point of view of photostability, to disperse PDI-O. It was concluded that the main photodegration mechanism of polymers doped with PDI-O was the chemical photodegradation due to oxygen (photooxidation).

${ }_{30}$ So, the good performance of PMMA was attributed to its low oxygen permeability.

\section{INSERT FIG. 1}

${ }_{35}$ Within this context, the general aim of this work is to improve even further the laser performance, in terms of threshold and photostability, of devices based on PDI-doped polymer films. For such purpose, we have first investigated the ASE properties of PDI-O, when doped into PS, and compare them to those of PDI${ }_{40}$ C6-doped PS films. Results have been interpreted by considering the absorption and photoluminescence (PL) properties of both derivatives, in solution and in thin film. Nuclear magnetic resonance (NMR) experiments in solution have also been performed, in order to get insights on the role of molecular 45 conformation on the optical properties of the films. A second aim of the work was to replace PS by PMMA, recently highlighted as one of the best matrixes for PDI-O, and compare their ASE results to those obtained with PS. The final step was to fabricate a 1D second-order DFB device using the best performing material, ${ }_{50}$ among the ones investigated, and compare its properties to that of DFBs based on PDI-C6-doped PS.

\section{Experimental section}

Materials and characterization methods.

${ }_{55}$ All chemicals were reagent-grade, purchased from commercial sources and used as received unless otherwise specified. PDI-C6 $(\mathrm{M}=755 \mathrm{~g} / \mathrm{mol})$ and PDI-O $(\mathrm{M}=711 \mathrm{~g} / \mathrm{mol})$ were purchased from LambdaChem and Phiton, respectively, with a purity higher than $99.5 \%$. PS $\left(\mathrm{M}_{\mathrm{w}}=35000 \mathrm{~g} / \mathrm{mol}\right)$ and PMMA $\left(\mathrm{M}_{\mathrm{w}}=15000\right.$ ${ }_{60} \mathrm{~g} / \mathrm{mol}$ ) were purchased from Sigma Aldrich. ${ }^{1} \mathrm{H}$ and ${ }^{13} \mathrm{C}$ NMR spectra were recorded on a Bruker AV300 and the chemical shifts were reported relative to tetramethylsilane (TMS) at $0.0 \mathrm{ppm}\left({ }^{1} \mathrm{H}\right.$ $\mathrm{NMR})$ and to $\mathrm{CDCl}_{3}$ at $77.16 \mathrm{ppm}\left({ }^{13} \mathrm{C} \mathrm{NMR}\right)$.

\section{Sample preparation}

${ }_{65}$ The organic active films were prepared by the spin-coating technique. Toluene solutions containing an inert polymer (PS or PMMA), doped with a varying amount (up to around $3 \mathrm{wt} . \%$ with respect to the polymer) of either PDI-C6 or PDI-O, were prepared and cast over $\mathrm{SiO}_{2}$ substrates. After deposition, films were heated 70 at $90{ }^{\circ} \mathrm{C}$ for $120 \mathrm{~min}$ in order to eliminate the residual solvent. The percentage of polymer with respect to the solvent was adjusted in order to obtain film thickness $(h)$ of around $600 \mathrm{~nm}$ and $1250 \mathrm{~nm}$ for PS and PMMA, respectively. These $h$ values, measured by means of an interferometer coupled to an optical 75 microscope, were chosen because they are just below the cut-off thickness for the propagation of the first high-order waveguide mode. So, the films support only one highly confined mode (the fundamental transverse electric mode, $\mathrm{TE}_{0}$ ), thus allowing to obtain the lowest thresholds. ${ }^{17}$ The $h$ values selected for PS and 80 PMMA are significantly distinct because their refractive indexes (n) are different (1.591 and 1.492 , at $\lambda=579 \mathrm{~nm}$, respectively). Films had good optical quality (transparency and homogeneity) and were stable for many months, when stored at room temperature in air and in the dark. For the ASE experiments, flat 85 and transparent fused silica substrates were used, while for DFB laser fabrication, the organic films were deposited on $\mathrm{SiO}_{2}$ films (grown by thermal oxidization of silicon wafers) over which surface relief gratings were previously engraved by thermal nanoimprint lithography and subsequent reactive ion etching. For 90 both types of substrates the refractive index was 1.46 (at $\lambda=579$ $\mathrm{nm})$.

Details on the DFB grating fabrication procedure and their characterization by field emission scanning electron microscopy and atomic force microscopy have been published elsewhere. ${ }^{15}$ 95 The resulting gratings, with an overall size of $(2 \times 2) \mathrm{mm}^{2}$, had equal line and space, a period $\Lambda$ of $368 \mathrm{~nm}$ and a depth $(d)$ of 125 nm.

\section{Optical experiments.}

Absorption and PL measurements were carried out in a Jasco V100650 spectrophotometer and a Jasco FP-6500/6600 fluorimeter, respectively. Experiments in solution were performed in the same solvent used for the spin-coating process (toluene) in quartz cells of $10 \times 10 \mathrm{~mm}^{2}$. The molar extinction coefficients $(\varepsilon)$ at the wavelength of maximum absorption $\left(\lambda_{\max }=527 \mathrm{~nm}\right)$ were 105 obtained from the linear fit of the optical density at that wavelength as a function of the PDI concentration in the solvent, that was varied between $2.4 \times 10^{-7}$ and $3 \times 10^{-5} \mathrm{M}$. The excitation wavelength to measure PL was $491 \mathrm{~nm}$, instead of the $533 \mathrm{~nm}$ beam used in the ASE and DFB experiments, in order to reduce 110 the overlap between absorption and emission. PL spectra for the films were obtained by exciting at a $60^{\circ}$ angle with respect to the 
normal to the film. PL emission was collected in reflection at a $30^{\circ}$ angle, in order to avoid the pump beam.

The ASE properties of the films deposited over substrates without 5 gratings were explored by optical excitation with a frequencydoubled Nd:YAG (YAG-yttrium aluminum garnet) laser (10 ns, $10 \mathrm{~Hz}$ ) operating at $532 \mathrm{~nm}$, which lies close to the maximum absorbance of PDI derivatives substituted at the $\mathrm{N}$ positions. ${ }^{11} \mathrm{~A}$ scheme of the experimental setup can be found elsewhere. ${ }^{28}$ The 10 energy of the pulses was varied using neutral density filters. The pump laser beam was expanded, collimated and only the central part was selected in order to ensure uniform intensity. A cylindrical lens and an adjustable slit were then used to shape the beam into a stripe of $3.5 \mathrm{~mm}$ by $0.53 \mathrm{~mm}$. The stripe was placed

15 right up to the edge of the film, from were PL emission was collected with an Ocean Optics USB2000-UV-VIS fiber spectrometer with 600 grating lines and a resolution in determining the emission linewidth of $1.3 \mathrm{~nm}$. The precision in measuring the emission wavelength was around half of this value.

20 The ASE photostability was determined by studying the time evolution of the ASE intensity, while the sample was excited in the same region, at constant pump intensity and under ambient conditions. The parameter used to characterize this property has been the same used in previous works from our group, ${ }^{11,12,14}$ i.e.

${ }_{25}$ the photostability halflife $\left(\tau_{1 / 2}\right)$, defined as the time (or number of pump pulses) at which the ASE intensity decays to half of its initial value.

The experimental setup to characterize the laser emission 30 properties of the DFB devices differs from the one used to measure ASE only in the geometrical configuration to excite the sample and to collect the emitted light. In particular, the cylindrical lens was replaced by a spherical one, so the beam over the sample (incident at $\sim 20^{\circ}$ with respect to the normal to the 35 film plane) was shaped into an elliptical spot (instead of a stripe) with a minor axis of $1.1 \mathrm{~mm}$. The emitted light was collected in a direction perpendicular to the film surface (instead of from the edge), by placing the fiber spectrometer at $1 \mathrm{~cm}$ from the sample.

\section{Results and discussion}

\section{ASE properties of PDI-doped PS films}

The absorption, PL and ASE properties of films doped with 0.5 wt.\% of PDI-C6 have been recently reported. ${ }^{17}$ In that work, the aim was to investigate the effect of changing film thickness on the ASE performance. In the present work, we have studied the 45 effect of varying the PDI concentration. For low doping ratios (below 0.5 wt.\%), the absorption, PL and ASE spectra of PDI-C6, were similar to those reported in ref. [17] for the film containing 0.5 wt. $\%$. As discussed in detail in the following section, for larger concentrations, the PL spectrum changed, due to the 50 formation of aggregated species. As a consequence, PL emission quenched and the ASE threshold increased. The behavior of PDIC6 is similar to that of PDI-N, ${ }^{12}$ given that they only differ in the length of the chains attached to the imide $\mathrm{N}$ positions. Concerning PDI-O, whose properties when doped into PS films 55 have not been previously reported, the shape of the absorption, PL and ASE spectra are also similar to those obtained when doping with small percentages of PDI-C6 (see Fig. 2). As for PDI-C6 (and PDI-N), in this case ASE takes place at a wavelength close to the peak of the $0-1$ PL transition. On the 60 other hand, changes in the PL spectrum, similar to those observed at high concentrations in the films containing PDI-C6, have not been observed for films doped with PDI-O. Moreover, these latter ones show larger PL efficiencies, which result in lower ASE thresholds $\left(I_{\text {th-ASE }}\right)$, as discussed below.

\section{INSERT FIG. 2}

The $I_{\text {th-ASE }}$ values have been obtained from plots of the emission linewidth, defined as the full width at half of its maximum 70 (FWHM), as a function of pump intensity (see Fig. 3(a)). In particular $I_{\text {th-ASE }}$ is defined as the pump intensity at which the FWHM decays to half of its maximum value. The existence of gain, results not only in a spectral narrowing at the threshold, but also in a considerable increase of the output intensity, as also 75 shown in Fig. 3 (a). It is remarkable the lower threshold obtained for the film doped with PDI-O, as compared to that containing PDI-C6. This result is applicable for a wide range of PDI concentrations, not only for 0.5 wt.\% (see Fig. 3(b)). For PDI-C6, the lowest threshold $\left(\sim 12 \mathrm{~kW} / \mathrm{cm}^{2}\right)$ has been obtained for the 80 film doped with 0.5 wt. $\%$. At higher concentrations, the threshold increased rapidly, reaching a value of $100 \mathrm{~kW} / \mathrm{cm}^{2}$ for doping ratios of $2.5 . \%$. On the other hand, for PDI-O the minimum threshold $\left(2-3 \mathrm{~kW} / \mathrm{cm}^{2}\right)$ was obtained for a range of concentrations between 0.75 and 3 wt.\%. For higher 85 concentrations, results were not reproducible due to limitations in the solubility of the compound.

\section{INSERT FIG. 3}

90 The ASE wavelength $\left(\lambda_{\text {ASE }}\right)$ for the films prepared varied between 577 and $581 \mathrm{~nm}$, depending on the doping ratio (see Fig. $\mathrm{S} 1$ (a) in the supporting information). $\lambda_{\mathrm{ASE}}$ increases with concentration, but only slightly (up to around $4 \mathrm{~nm}$ ), because the range of PDI concentrations is small. Within experimental 95 uncertainty, both PDI derivatives behave similarly. In other materials in which the concentration of active material could be varied in a wider range, much larger shifts in $\lambda_{\mathrm{ASE}}$ were obtained. ${ }^{29}$

The ASE linewidth $\left(\mathrm{FWHM}_{\mathrm{ASE}}\right)$, measured well above the ASE 100 threshold, also depends on the doping ratio (see Fig. S1(b) in the supporting information), although changes are small, again due to the range of concentrations explored. The $\mathrm{FWHM}_{\mathrm{ASE}}$ decreases gradually for both derivatives, reaching minimum values of around 4 and $6 \mathrm{~nm}$, for PDI-C6 and PDI-O respectively. 105 Nevertheless, optimizing this parameter is not so critical, since the emission linewidth of the lasers will be defined by the laser cavity.

Concerning the ASE photostability, $\tau_{1 / 2}$ values for PS films doped with different concentrations of both, PDI-C6 and PDI-O, are 110 listed in Table 1. For a proper comparison of different materials, measurements should be done at the same pump intensity $\left(I_{\text {pump }}\right)$ and doping ratio. In previous works with PS films doped with PDI-N and PDI-C6, we showed that the photostability depends 
strongly on the pump intensity. ${ }^{12,14}$ In those studies, ASE halflifes of various hundreds of minutes were obtained, when exciting two times above threshold. In the present work $I_{\text {pump }}$ was around 25 $\mathrm{MWcm}^{-2}$ in all cases (various orders of magnitude larger than the 5 corresponding ASE thresholds). The extreme pumping condition used here, that result in $\tau_{1 / 2}$ values of only a few minutes, were chosen for the practical purpose of shortening the length of the experiments. In the present study, our aim is to compare both derivatives and not to demonstrate the large photostability 10 lifetimes of these materials, which was already done. ${ }^{12,14}$

By comparing the halflives of PS films with similar film thickness (around $600 \mathrm{~nm}$ ) and same doping ratio (0.5 wt.\%), but using different PDI derivative, we see that the photostability of 15 PDI-O in this matrix is around 3 times larger that that of PDI-C6. Results also show that the effect of increasing the doping ratio in the PDI-O-doped PS films seems to have a negative effect, the largest $\tau_{1 / 2}$ value being obtained for a concentration of $0.5 \mathrm{wt} . \%$. As already mentioned, these experiments were done at the same 20 pump intensity, while the thresholds for the PDI-O-doped films are lower than those of films containing PDI-C6. This means that from a practical point of view, the performance of PDI-O in terms of operational device lifetime would be even better than that shown by these data, particularly when operating at moderate 25 pumping conditions (for example two times above the corresponding threshold in both cases).

Table 1 ASE performance of polymer films doped with PDI derivates.

\begin{tabular}{cccccc}
\hline $\begin{array}{c}\text { Polyme } \\
\mathrm{r}\end{array}$ & $\begin{array}{c}\text { PDI } \\
\text { derivativ } \\
\mathrm{e}\end{array}$ & $\begin{array}{c}{[\mathrm{PDI}]^{a} /} \\
\mathrm{wt.} \%\end{array}$ & $h^{b} / \mathrm{nm}$ & $\begin{array}{c}I_{\text {thASE }}{ }^{c} / \\
\mathrm{kW} / \mathrm{cm}^{2}\end{array}$ & $\begin{array}{c}\tau_{1 / 2}{ }^{d} / \mathrm{min} \\
\left(\times 10^{3} \text { pulses }\right)\end{array}$ \\
\hline PS & PDI-C6 & 0.5 & 610 & 12 & $10(6)$ \\
PS & PDI-O & 0.5 & 630 & 4 & $38(23)$ \\
PS & PDI-O & 1 & 600 & 3 & $12(7.2)$ \\
PS & PDI-O & 2 & 630 & 3 & $13(7.8)$ \\
& & & & & \\
PMMA & PDI-C6 & 0.5 & 1330 & 115 & $3(1.8)$ \\
PMMA & PDI-O & 0.5 & 1220 & 7 & $18(11)$ \\
PMMA & PDI-O & 1 & 1320 & 12 & $17(10)$
\end{tabular}

${ }^{a} \mathrm{PDI}$ concentration; ${ }^{b}$ Films thickness (error is around $5 \%$ ); ${ }^{c} \mathrm{ASE}$ threshold (error is around $10 \%$ ); Photostability halflife (error is around $10 \%$ ), measured under excitation at $2560 \mathrm{~kW} / \mathrm{cm}^{2}$.

\section{Absorption and photoluminescence properties of PDIs in solution and in PS films}

In order to understand the reason why the ASE thresholds of PS films doped with PDI-O are lower than those containing PDI-C6, we have studied in detail the absorption and PL properties. First 40 of all, we performed experiments in diluted solutions that typically provide information about the properties of the independent molecules. Then, we analyze these properties in the films, in which the effect of molecular interactions can play a role.

The absorption and PL properties of both PDI derivatives in solution are very similar to those previously reported for PDI-N. ${ }^{11}$ No changes in the spectral shape have been observed for the range of concentrations explored. As expected for this type of ${ }_{50}$ PDIs substituted in the imide nitrogen positions, large $\varepsilon$ values ( $60000 \mathrm{M}^{-1} \mathrm{~cm}^{-1}$ ) and high PL quantum yields (close to 1) were obtained for both derivatives. The fact that the optical absorption and the PL intensity increased linearly with concentration is indicative that no aggregation effects were present.

55

Concerning the absorption spectra of the films, for the range of concentrations explored, results were also similar to those reported for PDI-N dispersed in $\mathrm{PS}^{11}$ and no significant differences were found when comparing PDI-O and PDI-C6.

${ }_{60}$ With respect to the PL spectra, shown in Fig. 4, the situation is different. For films doped with PDI-C6 (see Fig. 4(a)), the shape of the PL spectra and the evolution with the PDI concentration are similar to those observed previously with PDI-N. ${ }^{11}$ For low PDI-C6 concentrations ( 0.5 wt.\%), the PL spectrum is similar to 65 that obtained in solution (it consists of a main band at $536 \mathrm{~nm}$ and a first vibrational peak at $576 \mathrm{~nm}$ ). On the other hand, when the PDI-C6 concentration is increased, the relative intensities of the various peaks conforming the spectrum change gradually until the intensity of the vibrational peak dominates. As for PDI-N, this 70 behavior is attributed to the formation of aggregated species. Another observation that supports this interpretation is the saturation of the total PL intensity when the concentration increases (see Fig. 5). On the other hand, for films containing PDI-O, the shape of the PL spectra practically do not change with 75 concentration as shown in Fig. 4(b). The relative intensities of the PL peaks change with concentration but very slightly. This indicates that the formation of aggregated species for PDI-O starts at a larger concentration than for PDI-C6. This is confirmed by the saturation of the PL intensity observed in Fig. 5 at 80 concentrations above $3 \mathrm{wt} . \%$. It is remarkable the significantly larger PL intensity obtained for the PDI-O films, which is the key to explain the considerably lower ASE thresholds obtained with these materials.

\section{${ }_{85}$ INSERT FIG. 4}

\section{INSERT FIG. 5}

\section{NMR experiments to get insights on the role of molecular} 90 conformation on the optical properties of the films

The aim of this section is to understand the reason why the formation of non-emitting aggregated species, which results in a decrease of the PL intensity and therefore in an increase of the ASE threshold, appears for PDI-O at higher concentrations than ${ }_{95}$ for PDI-C6. For such purpose, we first performed ${ }^{1} \mathrm{H}$ and ${ }^{13} \mathrm{C}$ NMR experiments in solution in order to determine, in combination with previously published MM2 calculations, the molecular conformation of each derivative. Note that in dilute solution, PDIs are isolated, while in concentrated solutions, PDIs 100 form aggregated states due to intermolecular interactions. The attractive intermolecular forces depend, among other factors, on the steric hindrance caused by the substituents. Therefore, it is important to know the conformation of each derivate and how it affects the interaction among the aromatic PDI cores of 
neighbouring molecules.

${ }^{1} \mathrm{H}$ and ${ }^{13} \mathrm{C}$ NMR experiments of PDI-C6 and PDI-O in solution at room temperature were performed. The spectra are displayed in Figs. S2 and S3 of the supporting information. Data extracted 5 from them are listed below.

\section{N,N'-di(1-hexylheptyl)perylene-3,4:9,10-tetracarboxylic diimide (PDI-C6)}

${ }^{1} \mathrm{H}$ NMR (300 MHz, $\left.\mathrm{CDCl}_{3}, \delta\right): 0.83(\mathrm{t}, 12 \mathrm{H}), 1.27(\mathrm{~m}, 32 \mathrm{H})$, $101.86(\mathrm{~m}, 4 \mathrm{H}), 2.24(\mathrm{~m}, 4 \mathrm{H}), 5.19(\mathrm{~m}, 2 \mathrm{H}), 8.61(\mathrm{~d}, 4 \mathrm{H}), 8.66(\mathrm{br}$ d, $4 \mathrm{H}) .{ }^{13} \mathrm{C}$ NMR $\left(300 \mathrm{MHz}, \mathrm{CDCl}_{3}, \delta\right): 14.06,22.59,26.94$, 29.23, 31.77, 32.38, 54.78, 123.04, 123.10, 124, 126.47, 129.61, $131.15,131.91,134.52,163.60,164.66$.

\section{N,N'-di(2,6-diisopropylphenyl)-3,4:9,10-tetracarboxylic}

\section{5 diimide (PDI-O)}

${ }^{1} \mathrm{H}$ NMR (300 MHz, $\mathrm{CDCl}_{3}, \delta$ ): 1.18 (d, 24H), 2.76 (septet, 4H), $7.36(\mathrm{~d}, 4 \mathrm{H}), 7.51(\mathrm{t}, 2 \mathrm{H}), 8.71(\mathrm{~d}, 4 \mathrm{H}), 8.77(\mathrm{~d}, 4 \mathrm{H}) .{ }^{13} \mathrm{C}$ NMR $\left(300 \mathrm{MHz}, \mathrm{CDCl}_{3}, \delta\right.$ ): 23.39, 28.60, 122.73, 122.79, 123.51, 126.22, 126.41, 129.09, 129.56, 129.87, 131.13, 131.49, 133.58, $20134.45,145.01,162.86,163.59$.

Concerning the ${ }^{1} \mathrm{H}$ NMR spectrum of PDI-C6, two different signals (at 1.9 and $2.2 \mathrm{ppm}$ ), associated to the eight methylene hydrogens $\beta$ (second neighbours), relative to the $\mathrm{N}$ atoms, are 25 seen. The reason for observing two different signals is attributed to the fact that the two hygrogens in each $\beta$ methylene are diastereotopic. The signal observed at $5.2 \mathrm{ppm}$, due to the $\alpha$ hydrogen (relative to the $\mathrm{N}$ atom), is indicative of the existence of an intramolecular hydrogen bond between the $\alpha$ hydrogen and the 30 oxygen. This signal is considerably deshielded compared to the expected aliphatic region of 0-3 ppm. The hydrogen bond forces the alkyl chains to acquire the conformation shown in Fig. 6(a). In order to simplify the structure of the molecule in the scheme, the peripheral aliphatic chains have been replaced by methyl

35 groups and only one of the two possible diastereomers (due to rotation of the $\mathrm{C}-\mathrm{N}$ bond) is shown. This conformation is confirmed by the ${ }^{13} \mathrm{C}$ NMR spectrum, which shows two carbonyl signals at 163.6 and $163.7 \mathrm{ppm}$, due to the asymmetric environment of the carbonyl groups. This asymmetry affects to 40 other carbons, such as $\mathrm{C}_{4}$ and $\mathrm{C}_{5}$ (see Fig. 1(a)), which also show two signals at 123.1 and $124.0 \mathrm{ppm}$ (for $\mathrm{C}_{4}$ ) and at 131.2 and 131.9 (for $\mathrm{C}_{5}$ ). The NMR results obtained for PDI-C6 are analogous (taking into account the different length of the alkyl chains attached to the $\mathrm{N}$ atoms) to those previously reported for ${ }_{45}$ PDI-N. ${ }^{30}$ In that work the NMR spectra were interpreted as a result of restricted rotation around the imide $\mathrm{C}-\mathrm{N}$ bond. Through MM2 calculations, the lowest energy conformation was found at $0^{\circ}$ rotation, i.e. one carbonyl located between the alkyl chains and the other one eclipsed by the $\alpha$ hydrogen.

${ }_{50}$ With regards to PDI-O, the ${ }^{13} \mathrm{C}$ NMR spectrum (Fig. S3(b) in supporting information) shows two signals at 23.4 and $28.6 \mathrm{ppm}$, associated to the $\mathrm{C}_{8}$, and $\mathrm{C}_{7}$, carbons, respectively. The signal at $162.9 \mathrm{ppm}$ corresponds to the carbonyl groups. There is also a small signal at $163.6 \mathrm{ppm}$ that might indicate the existence of an 55 asymmetric environment for these carbonyl groups. The optimized ground state geometry for PDI-O, determined by density functional theory (DFT), evidences that the 2,6- diisopropylphenyl groups form angles of $89.5^{\circ}$ with respect to the flat aromatic PDI core. ${ }^{31}$ Due to this essentially perpendicular 60 conformation, it could be expected that the four carbonyl groups would be equivalent. However, for DXP perylene, whose chemical structure is very similar to that of PDI-O (the only difference is that the substituents attached to the $\mathrm{C}_{2}$, and $\mathrm{C}_{6}$, carbons in the phenyls - see Fig. 1 (b) - are methyl groups, ${ }_{65}$ instead of isopropyl groups), the ground state energy wells are flat around the minima ${ }^{32,33}$ and have a global minimum at $90^{\circ}$ rotation around the phenyl-N bond, which is only $200 \mathrm{~cm}^{-1}$ deeper than the energies at $65^{\circ}$ and $115^{\circ}$ rotation. ${ }^{34}$ This energy barrier is on the order of the thermal energy at room temperature, 70 so favouring fluctuations of the ground state conformation. If this situation occurs also for PDI-O, that would explain the small ${ }^{13} \mathrm{C}$ NMR signal observed at $163.6 \mathrm{ppm}$. The existence of conformers for PDI-O, with different ground-state twist angles, has been reported in sol-gel thin films. ${ }^{35}$ The lowest energy conformation 75 for PDI-O is shown in Fig. 6(b).

When PDIs are not isolated, i.e. when they are dispersed in matrixes at sufficiently large concentrations, their emission properties might be considerably affected by aggregation effects, which depend strongly on the type of conformation of the 80 substituents attached to the PDI core. Taking into account the NMR results previously described, the relationship between the conformation as well as the steric hindrances of the substituents and the interaction among the aromatic PDI cores of neighbouring molecules can be analyzed. This helps 85 understanding the reason why the formation of aggregated species in films doped with PDI-O starts at a larger concentration than for those containing PDI-C6. The discussion has been done by considering also previously reported experimental $\mathrm{x}$-ray data of single crystals based on these PDI derivatives or others with 90 analogous chemical structure. ${ }^{32,36-38}$

The influence on colour crystal of the type of crystal packing was investigated by Kazmaier et al. ${ }^{39}$ for various PDIs with different substituents at the imide $\mathrm{N}$ positions. They observed that small changes in the substituents lead to large changes in the colour of 95 the crystals. The steric requirements of the substituents caused a transverse and longitudinal offset between two neighbouring PDI molecules in order to avoid unfavourable interactions between their cores. In crystals based on PDIs with ethyl groups attached to the $\mathrm{N}$ atomos, other authors have observed a relative twisting 100 between molecules along the stacking direction. ${ }^{37}$ This twisting was computed in the isolated dimer model for a PDI derivative with 1-heptyloctyl groups attached to the imide $\mathrm{N}$ imide (denoted as $\mathrm{C}_{8,7} \mathrm{PDI}$ ), obtaining a twist angle of $35^{\circ}$ and a stacking distance in the range $3.4-3.5 \AA^{40}$ We suggest that the 105 aggregated species forming in our PDI-C6-based films may adopt a conformation similar to that of the $\mathrm{C}_{8,7}$-PDI dimmer. In fact, stacking distances in the range $3.4-3.5 \AA$ appear in most planar perylene structures known. ${ }^{39}$ In constrast, for the DXP perylene, a larger stacking distance of $6.65 \AA$ or a pronounced transverse 10 offset shift was found. ${ }^{37}$ That was explained by the near perpendicular orientation of the 2,6-methylphenyl groups, which difficults the overlapping of the aromatic PDI cores. Both, PDIDXP and PDI-O, have phenyl groups attached to the $\mathrm{N}$ atoms and in both cases the phenyls have substitutions at the same carbons 
$\left(\mathrm{C}_{2}\right.$, and $\mathrm{C}_{6}$, see Fig. 1(b)). In general, the stacking distance and therefore, the interaction of neighbouring aromatic PDI cores, is affected by the steric hindrances of the groups attached to the phenyls, which might depend significantly on the particular 5 positions at which these are located. For example, for the PDI denoted as PR149, which differs from PDI-DXP only in the phenyl positions at which the methyl groups are attached $\left(\mathrm{C}_{3}\right.$, and $\mathrm{C}_{5}$, , see Fig. 1(b)), ${ }^{41}$ the stacking distance is $3.46 \AA^{42}{ }^{42}$ This is in contrast with the stacking distance of $6.65 \AA$ measured for PDI-

10 DXP. In other PDIs with phenyls attached to the $\mathrm{N}$ positions, in which the phenyls are substituted at the $\mathrm{C}_{2}$, and $\mathrm{C}_{6}$, carbons with $\mathrm{F}$ atoms and at the $\mathrm{C}_{3}$, and $\mathrm{C}_{5}$, carbons with $\mathrm{Cl}$ atoms, the stacking distances are in the range 3.43-3.50 $\AA .{ }^{43}$ In these latter PDI derivatives, the steric hindrances of the groups attached to 15 the phenyls and the final molecular conformations are different than that of PDI-DXP. Given that PDI-O and PDI-DXP have a similar conformation, it is expected that their stacking distance and their transverse offset would be similar. Finally, considering that the conformation of neighbouring molecules for both PDI-C6 20 and PDI-O in PS films may be similar to the crystal structure conformation, we suggest that the higher PL efficiency of films containing PDI-O relative to those doped with PDI-C6, is due to the lower interaction between aromatic PDI cores, caused by the higher steric requirements of the 2,6-diisopropylphenyl groups of 25 the PDI-O.

\section{INSERT FIG. 6}

The results obtained in this section provide useful general 30 guidelines to choose (or to design) PDI derivatives with good ASE properties when dispersed in polymer films. The main idea is that the groups attached to $\mathrm{N}$ atoms should avoid the interaction between neighbouring aromatic PDI cores. The strategies used in our case, that had lead to reach that end, and 35 that might be somewhat generalized are: (i) use a rigid group with an essentially perpendicular conformation with respect to the PDI core, as the phenyl groups of PDI-DXP and PDI-O; (ii) the positions at which additional substituents are added to the groups attached to the $\mathrm{N}$ atoms should be near the PDI core. This occurs

40 in PDI-O and PDI-DXP, in which the isopropyl and methyl groups respectively, are attached at $\mathrm{C}_{2}$, and $\mathrm{C}_{6}$, carbons (see Fig. 1(b)); and (iii) these substituents added to the groups attached to the $\mathrm{N}$ atoms should have large steric hindrances.

\section{${ }_{45}$ Role of the matrix on the ASE performance: PS versus PMMA}

In our previous works on lasers based on dye-doped polymer active films, we used PS as a matrix. This polymer has a high refractive index and a good transparency, thus allowing to obtain 50 waveguides with good mode confinement and low propagation losses. These parameters have an important influence on the thresholds, as discussed in detail in ref. [17]. Here, we do not intend to perform a systematic study of different matrixes, but to compare PS to PMMA, which is a material often used for lasing 55 applications as discussed in the introduction.

We prepared several samples using PMMA as a matrix, instead of PS, and characterized their ASE properties. Results are listed in Table 1. Thresholds shown in Table 1 are in all cases lower for the PS films than for the PMMA ones. For those doped with ${ }_{60} \mathrm{PDI}-\mathrm{C} 6$ the difference is quite larger (around 10 times), while it is smaller in the case of PDI-O (2-4 times depending on the doping ratio). With regards to the photostability, by comparing the halflives measured under the same pump intensity, we see that the performance is sligtly better for PS. This is contradictory with the ${ }_{65}$ idea proposed by other authors that oxygen permeability is the most important factor in determiming the photostability of PDIO-doped films, ${ }^{27}$ given that the oxygen permeability of PS $(2.53$ barrers, at $\left.25^{\circ} \mathrm{C}\right)^{44}$ is around 15 times larger than that of PMMA $\left(0.16\right.$ barrers at $\left.30^{\circ} \mathrm{C}\right)$. Therefore, our results suggest that there 70 are other parameters that have an important role in the photostability. In fact, in previous works dealing with the photostability of PDI derivates diluted in several matrixes, different photodegradation mechanisms have been proposed: photochemical reactions of the PDIs with free radicals, 75 surrounding species, reactive hydrocarbons or impurities; photochemical reactions of the PDIs in their long-lived triplet state; multiphoton absorption ${ }^{45}$ or the presence of dark states from molecules with different molecular conformations. ${ }^{46}$ In the work of Tanaka et al., ${ }^{24}$ it was discussed that the 80 photodegradation of PDI-O in PMMA hosts was due two mechanisms: (i) type II photooxidation, that predominates under aerobic conditions, and (ii) partially reversible photoreduction, which prevails under anaerobic conditions. Their results indicated that oxygen-free device fabrication and encapsulation were not 85 suitable procedures for PDI-based optical devices, because the exclusion of oxygen lead to photoreduction. Therefore, in order to discuss the photostability results of our PS and PMMA films we should consider, not only the oxygen permeability of the matrix, but also the type and strength of the dye-polymer 90 interaction, among others factors. In the absence of oxygen, the photoreduction of the PDI molecules would be more favourable as the strength of electron donors of the groups of the polymer increase according to the series $-\mathrm{Cl}<-\pi<-\mathrm{COO}-<-\mathrm{CN}<-$ $\mathrm{CON}=<-\mathrm{N}=.^{47}$ The phenyl groups of PS have a lower electron 95 donor character than that of the-COO-groups of PMMA. As a consequence, possibly the photoreduction of the PDIs would be lower when dispersed in PS than in PMMA. On the other hand, in the presence of oxygen, the predominant photodegration mechanism would be type II photooxidation, although some 100 photoreduction could also be present. ${ }^{24}$ Oxygen permeability for PMMA is lower than that of PS, so photooxidation of the PDI molecules would be smaller when dispersed in PMMA. However, the electron donor character of PMMA is larger than that of PS, which would result in a higher photoreduction, as compared to 105 PS.

In summary, our results show that for PDI derivatives, PS is a better matrix than PMMA, in terms of both, threshold and photostability. Although the photostability results shown in Table 1101 (obtained at the same pump intensity) indicate that PS is just slightly better than PMMA, in practical devices (pumped at moderate conditions, i.e. two or three times above the corresponding threshold for each material), PS would be clearly superior than PMMA. This is because PS-based devices have 115 lower thresholds, so they can be operated at lower pump 
intensities than the PMMA ones.

\section{DFB lasers based on PDI-doped PS films.}

5 In this section we aimed to investigate whether the improved ASE performance of PDI-O (in comparison to PDI-C6) shown in previous sections can be extented to DFB lasers. For such purpose, we fabricated a DFB device (scheme shown in Fig. 7(a)) using PDI-O-doped PS as active material, and compared its laser 10 performance to that of previously reported PDI-C6-based devices with similar structure. ${ }^{14,15}$ In ref. [15] the emission wavelength was tuned from 554 to $583 \mathrm{~nm}$ by varying $h$ between 240 and $1200 \mathrm{~nm}$. For the particular device prepared here (with $h \sim 600$ $\mathrm{nm}$ and $d=125 \mathrm{~nm}$ ), DFB emission is associated to the $\mathrm{TE}_{0}$ 15 waveguide mode and occurs at around $576 \mathrm{~nm}$. Such film thickness has been chosen because it allows to minimize the DFB threshold and also because it would be independent on the grating depth. ${ }^{15}$

${ }_{20}$ Fig. 7(b) shows the emission spectra obtained under excitation below and above threshold for the PDI-O-based laser. From the point of view of spectral shape, results are similar to those obtained with devices based on PDI-C6 instead of PDI-O. ${ }^{15}$ As discussed in that work, the dip observed in the spectrum below 25 threshold is indicative that index coupling contributes to the gain process. Concerning the spectrum obtained above threshold, a single DFB peak appears at the long-wavelength edge of the Bragg dip. The observation of DFB emission outside the dip would be also an indication that index-coupling modulation is 30 contributing significantly to the gain mechanism, although the presence of gain coupling can not be excluded

\section{INSERT FIG. 7}

${ }_{35}$ The most important result is the significant decrease in the DFB threshold obtained when using PDI-O instead of PDI-C6 (see Fig. 8). As discussed in previous sections, this is due to the higher PL efficiency and lower ASE threshold of the films containing PDIO. This means that the already efficient PDI-based DFB lasers 40 previously reported, can be further improved by using PDI-O, instead of PDI-C6. Another important consequence of achieving a decrease in threshold is that the device operational lifetime would also improve, given that the pump intensity required for laser oscillation would be smaller.

\section{INSERT FIG. 8}

\section{Conclusions}

The performance of lasers based on polymer films doped with ${ }_{50}$ PDI derivatives as active media has been improved with respect to previous works. A key aspect for such improvement has been the use of a PDI compound (PDI-O) whose conformation leads to a distance among the cores of neighbouring molecules in the film larger than the one obtained with other PDIs. As a consequence 55 of the reduced molecular interaction, a larger PL efficiency and a lower ASE threshold is obtained. From the point of view of the polymer matrix used to disperse the PDIs, it was found that PS is superior to PMMA.

PS films doped with PDI-O have shown lower ASE thresholds 60 and larger photostabilities than those of films containing PDI-C6. For PDI-C6, the lowest threshold $\left(\sim 12 \mathrm{~kW} / \mathrm{cm}^{2}\right)$ was obtained for the film doped with 0.5 wt. $\%$, while it increased rapidly at lower or higher concentrations. On the other hand, for PDI-O the minimum threshold $\left(2-3 \mathrm{~kW} / \mathrm{cm}^{2}\right)$ was obtained for a wider range 65 of concentrations (between 0.75 and 3 wt.\%). The better ASE performance of the films containing PDI-O is due to their larger PL efficiency, which is attributed to the fact that non-emitting aggregated species appear at higher concentrations than for PDIC6. By performing NMR experiments, to elucidate the PDI 70 molecular conformation, and considering MM2 calculations and $\mathrm{x}$-ray data of these (or analogous) PDI derivatives available in the literature, we propose that the reason for having less aggregation with PDI-O is the higher steric requirements of its 2,6diisopropylphenyl groups. Concerning the ASE photostability of 75 PDI-doped PS films, it is around three times larger for PDI-O that for PDI-C6, when excited at the same pump intensity, and much larger if pumped at the same relative intensity with respect to their thresholds. The superiority of PDI-O in terms of ASE performance might be likely extended to a wider range of PDI 80 derivatives (not only with respect to PDI-C6), given that for most planar perylene structures, the distance between the cores of neighbouring PDI molecules is similar to that of PDI-C6 and therefore smaller than for PDI-O.

With regards to the matrix, PS films have shown lower thresholds 85 than the PMMA ones (around 10 and 3 times when using PDI-C6 and PDI-O, respectively) and larger photostabilities (only sligthly, when pumped at the same intensity, and clearly superior, when excited at the same relative intensity with respect to their thresholds). The fact that PS has a higher oxygen permeability 90 than PMMA is an indication that chemical photodegradation due to oxygen (photooxidation) is not the most important photodegradation mechanism for these materials. So, other factors, such as the type and strength of the dye-polymer interactions, should be considered.

${ }_{95}$ Finally, a second-order 1D DFB laser, using PDI-O-doped PS as active material, has been fabricated and its laser performance compared to that of previously reported PDI-C6-based devices with similar structure. We observed a significant decrease in the DFB threshold obtained when using PDI-O instead of PDI-C6, in 100 accordance with the lower ASE thresholds of the films.

\section{Acknowledgments}

We thank support from the Spanish Government (MECC) and the European Community (FEDER) through grants MAT200806648-C02 and MAT-2011-28167-C02, as well as to the 105 University of Alicante. MGR and MM are supported respectively by a CSIC fellowship within the program JAE and by MEC through a FPI fellowship. We also acknowledge V. Esteve for technical assistance.

\section{${ }_{110}$ Notes and references}

${ }^{a}$ Dpto. Física Aplicada, Instituto Universitario de Materiales de Alicante y Unidad Asociada UA-CSIC, Universidad de Alicante, 03080 Alicante, 
Spain.E-mail: maria.diaz@ua.es; Fax: +34 96590 9726; Tel: +34 96 5903543.

${ }^{b}$ Dpto. Óptica, Instituto Universitario de Materiales de Alicante y Unidad Asociada UA-CSIC, Universidad de Alicante, 03080 Alicante, Spain.

${ }_{5}^{c}$ Micro and Nanotechnology Department, IK4-Tekniker, Eibar 20600, Spain

$\dagger$ Electronic Supplementary Information (ESI) available: This section includes: Fig. S1 that shows the PDI conentration dependence of the ASE wavelength and linewidth; and Figs. S2 and S3 that show the NMR 10 spectra of PDI-C6 and PDI-O respectively. See DOI: 10.1039/b000000x/

1. N. Tessler, Adv. Mater., 1999, 11, 363.

2. M. D. McGehee and A. J. Heeger, Adv. Mater., 2000, 12, 1655.

3. I. D. W. Samuel and G. A. Turnbull, Chem. Rev., 2007, 107, 1272.

15 4. J. Clark and G. Lanzani, Nat. Photonics, 2010, 4, 438.

5. F. Hide, M. A. Díaz-García, B. Schwartz, M. Andersson, Q. Pei and A. J. Heeger, Science, 1996, 273, 1833.

6. N. Tessler, G. J. Denton and R. H. Friend, Nature, 1996, 382, 695.

7. Y. Yang, G. A. Turnbull and I. D. W. Samuel, Appl. Phys. Lett., 2008, 92, 163306.

8. M. Lu, S. S. Choi, C. J. Wagner, J. G. Eden and B. T. Cunningham, Appl. Phys. Lett., 2008, 92, 261502.

9. M. B. Christiansen, J. M. Lopacinska, M. H. Jakobsen, N. A Mortensen, M. Dufva and A. Kristensen, Opt. Express, 2009, 17, 2722.

10. M. D. McGehee, R. Gupta, S. Veenstra, E. M. Miller, M. A. DíazGarcía and A. J. Heeger, Phys. Rev. B, 1998, 58, 7035.

11. E. M. Calzado, J. M. Villalvilla, P. G. Boj, J. A. Quintana, R. Gomez, J. L. Segura and M. A. Díaz-García, J. Phys. Chem. C, 2007, 111, 13595

12. E. M. Calzado, J. M. Villalvilla, P. G. Boj, J. A. Quintana, R. Gómez, J. L. Segura and M. A. Díaz-García, Appl. Opt., 2007, 46, 3836.

13. M. A. Díaz-García, E. M. Calzado, J. M. Villalvilla, P. G. Boj, J. A. Quintana, F. J. Cespedes-Guirao, F. Fernandez-Lazaro and A. Sastre-Santos, Synth. Met., 2009, 159, 2293.

14. V. Navarro-Fuster, E. M. Calzado, P. G. Boj, J. A. Quintana, J. M. Villalvilla, M. A. Díaz-García, V. Trabadelo, A. Juarros, A Retolaza and S. Merino, Appl. Phys. Lett., 2010, 97, 171104.

15. V. Navarro-Fuster, I. Vragovic, E. M. Calzado, P. G. Boj, J. A. Quintana, J. M. Villalvilla, A. Retolaza, A. Juarros, D. Otaduy, S. Merino and M. A. Díaz-García, J. Appl. Phys., 2012, 112, 043104.

16. M. G. Ramirez, P. G. Boj, V. Navarro-Fuster, I. Vragovic, J. M. Villalvilla, I. Alonso, V. Trabadelo, S. Merino and M. A. DíazGarcía, Opt. Express, 2011, 19, 22443.

45 17. E. M. Calzado, M. G. Ramírez, P. G. Boj and M. A. Díaz García Appl. Opt., 2012, 51, 3287.

18. R. Reisfeld and G. Seybold, Chimia, 1990, 44, 295.

19. M. Canva, P. Georges, J. P. Perelgritz, A. Brun, F. Chaput and J. P. Boilot, Appl. Opt., 1995, 34, 428.

50 20. M. Faloss, M. Canva, P. Georger, A. Brun, F. Chaput and J. P. Boilot, Appl. Opt., 1997, 36, 6760.

21. M. D. Rahn, T. A. King, A. A. Gorman and I. Hamblett, Appl. Opt., 1997, 36, 5862.

22. A. K. Sheridan, A. R. Buckley, A. M. Fox, A. Bacher, D. D. C. Bradley and I. D. W. Samuel, J. Appl. Phys., 2002, 92, 6367.

23. Y. Yang, M. Wang, G. Qian, Z. Wang and X. Fan, Opt. Mater., 2004, 24, 621.

24. N. Tanaka, N. Barashkov, J. Heath and W.N. Sisk, Appl. Opt., 2006, 45, 3846 .

60 25. I. García-Moreno, A. Costela, M. Pintado-Sierra, V. Martín and R. Sastre, Opt. Express, 2009, 17, 12777.

26. A. Miasojedovas, K. Kazlauskas, G. Armonaite, V. Sivamurugan, S. Valiyaveettil, J. V. Grazulevicius and S. Jursenas, Dyes and Pigments, 2012, 92, 1285.

65 27. L. Cerdán, A. Costela, G. Durán-Sampedro, I. García-Moreno, M. Calle, M. Juan-y-Seva, J. de Abajo and G. A. Turnbull, J. Mater. Chem., 2012, 22, 8938

28. M. A. Díaz-García, E. M. Calzado, J. M. Villalvilla, P. G. Boj, J. A. Quintana, F. Giacalone, J. L. Segura and N. Martín, J. Appl. Phys., $2005,97,063522$.
29. E. M. Calzado, J. M. Villalvilla, P. G. Boj, J. A. Quintana and M. A. Díaz-García, Org. Electron., 2006, 7, 319.

30. L. D. Wescott and D. L. Mattern, J. Org. Chem., 2003, 68, 10058

31. A. Aldongarov, N. N. Barashkov and I. S. Irgibaeva, Int. J. Quantum Chem., 2007, 107, 2331.

32. E. Hädicke and F. Graser, Acta Crystallogr. , 1986, C42, 195

33. G. Klebe, F. Graser, E. Hädicke and J. Berndt, Acta Crystallogr., 1989, B45, 69

34. M. Sadraï, L. Hadel, R. R. Sauers, S. Husain, K. Krogh-Jespersen, J. D. Westbrook and G. R. Bird, J. Phys. Chem., 1992, 96, 7988

35. C. Julien, A. Débarre, D. Nutarelli, A. Richard and P. Tchénio, J. Phys. Chem., 2006, 110, 3902.

36. (a) F. Graser and E. Hädicke, Liebigs Ann. Chem., 1980, 12, 1994; (b) F. Graser and E. Hädicke, Liebigs Ann. Chem., 1984, 3, 483; (c)

85 E. Hädicke and F. Graser, Acta Crystallogr. Sect. C, 1986, 42, 189.

37. G. Klebe, F. Graser, E. Hädicke and J. Berndt, Acta Crystallogr. Sect. $B, 1989,45,69$.

38. P. Zugenmaier, J. Duff and T. L. Bluhm, Cryst. Res. Technol., 2000 35, 1095 .

90 39. P. M. Kazmaier and R. Hoffmann, J. Am. Chem. Soc., 1994, 116, 9684.

40. M. R. Hansen, R. Graf, S. Sekharan and D. Sebastiani, J. Am. Chem Soc., 2009, 131, 5251.

41. J. Mizuguchi and K. Tojo, J. Phys. Chem. B, 2002, 106, 767.

95 42. J. Mizuguchi and K. Tojo, Z. Kristallogr. NCS, 2001, 3, 37.

43. P. Zugenmaier, J. Duff and T. L. Bluhm, Cryst. Res. Technol., 2000, 35, 1095.

44. J. Brandrup, E. H. Immergut, and E. A. Grulke, Polymer Handbook, 4th Ed., Wiley-Interscience, New York, USA, 1999.

00 45. M. D. Rahn and T. A. King, Appl. Opt., 1994, 34, 1995.

46. C. Julien, A. Débarre, D. Nutarelli, A. Richard, and P. Tchénio, $J$ Phys. Chem. B, 2005, 109, 23145.

47. A.T. Slark and P.M. Hadgett, Polymer, 1999, 40, 1325. 


\section{Figure captions}

Figure 1.- Chemical structures of PDI-C6 (a) and PDI-O (b). The numbers indicate positions of the $\mathrm{C}$ atoms.

Figure 2.- Optical absorption (ABS, thin full line, left axis), 5 photoluminescence (PL, right axis, dashed line) and amplified spontaneous emission (ASE, right axis, thick full line) for a 1 wt.\% PDI-O-doped PS film.

Figure 3.- (a) Output intensity at $\lambda=579 \mathrm{~nm}$ (left axis, open symbols) and linewidth, FWHM (right axis, full symbols), as a 10 function of pump intensity for 0.5 wt.\% PDI-C6-doped and 1 wt.\% PDI-O-doped PS films (squares and triangles, respectively); (b) ASE thresholds, determined from (a) as the intensity at which the FWHM decays to half of its maximum value, versus PDI concentration, for films doped PDI-C6 or PDI-O. Lines 15 connecting points are guides to the eye.

Figure 4.- PL spectra of PS films doped with various amounts of PDI-C6 (a) and PDI-O (b).

Figure 5.- Total PL intensity (integrated area below the spectrum) for PS films doped with different concentrations of 20 PDI-C6 (full squares) or PDI-O (full triangles). Lines connecting points are guides to the eye.

Figure 6.- Schemes of the molecular conformation of isolated PDI-C6 (a) and PDI-O (b), as a result of NMR. For simplicity the side chains of PDI-C6 have been replaced by methyl groups. The 25 dashed line in (a) indicates a hydrogen bond.

Figure 7.- (a) Scheme of DFB laser ( $\Lambda$ : grating period; $d$ : grating depth; $h$ : thickness of active film); (b) Emission spectra above and below threshold (full and dashed lines respectively) for a DFB device based on PS doped with 1 wt.\% of PDI-O as active 30 laser material.
Figure 8.- Output intensity versus excitation pump energy for DFB lasers based on PS doped with 0.5 wt.\% of PDI-C6 or with 1 wt.\% of PDI-O. 


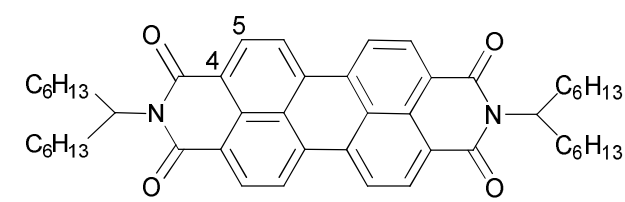

(a)

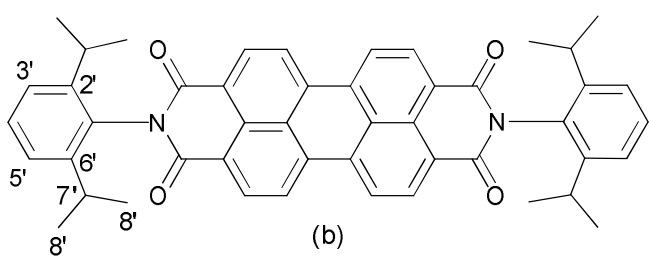

Fig. 1. 


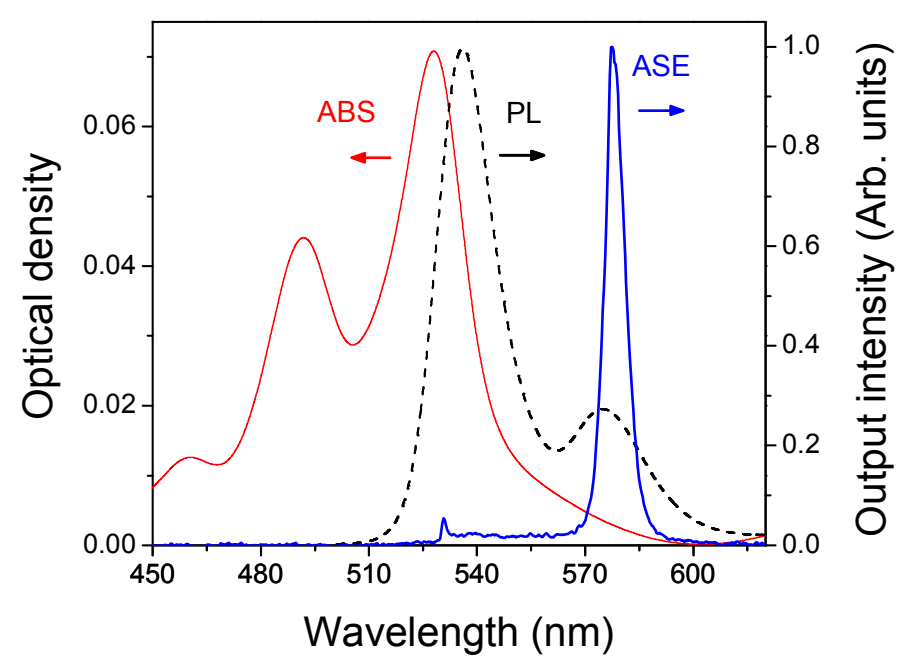

Fig. 2. 

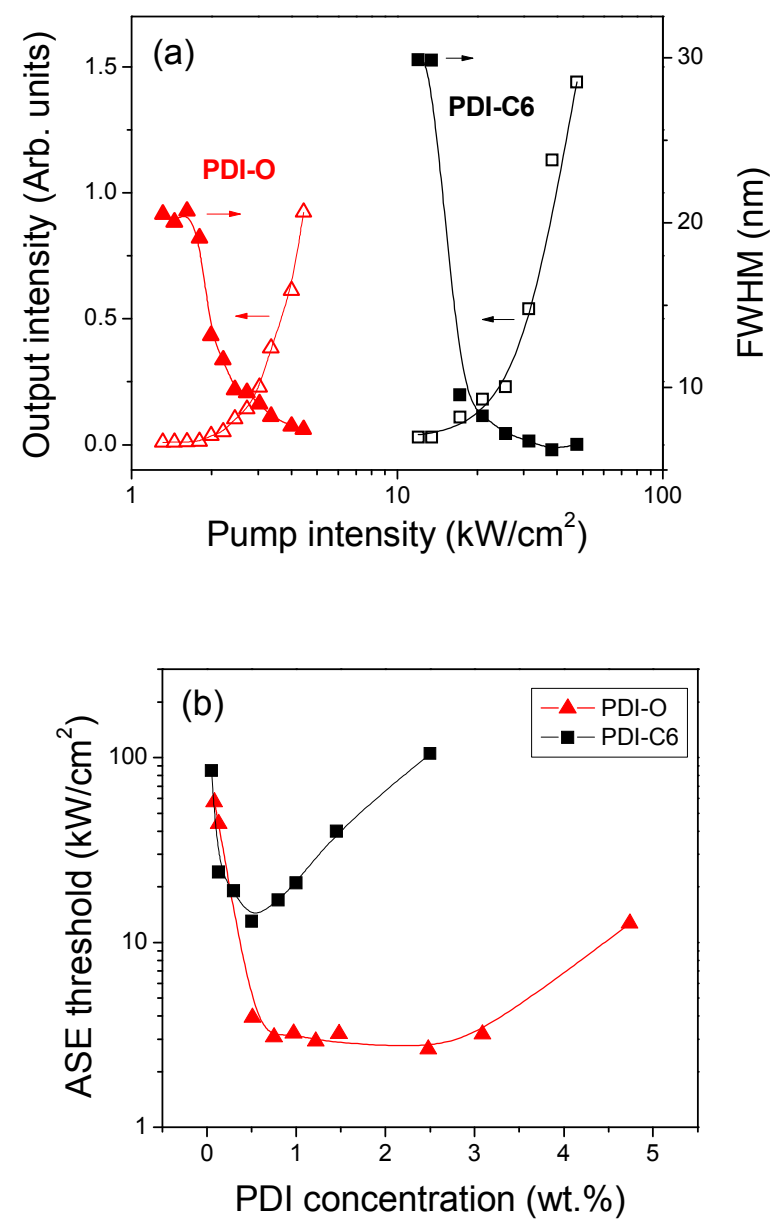

Fig. 3 

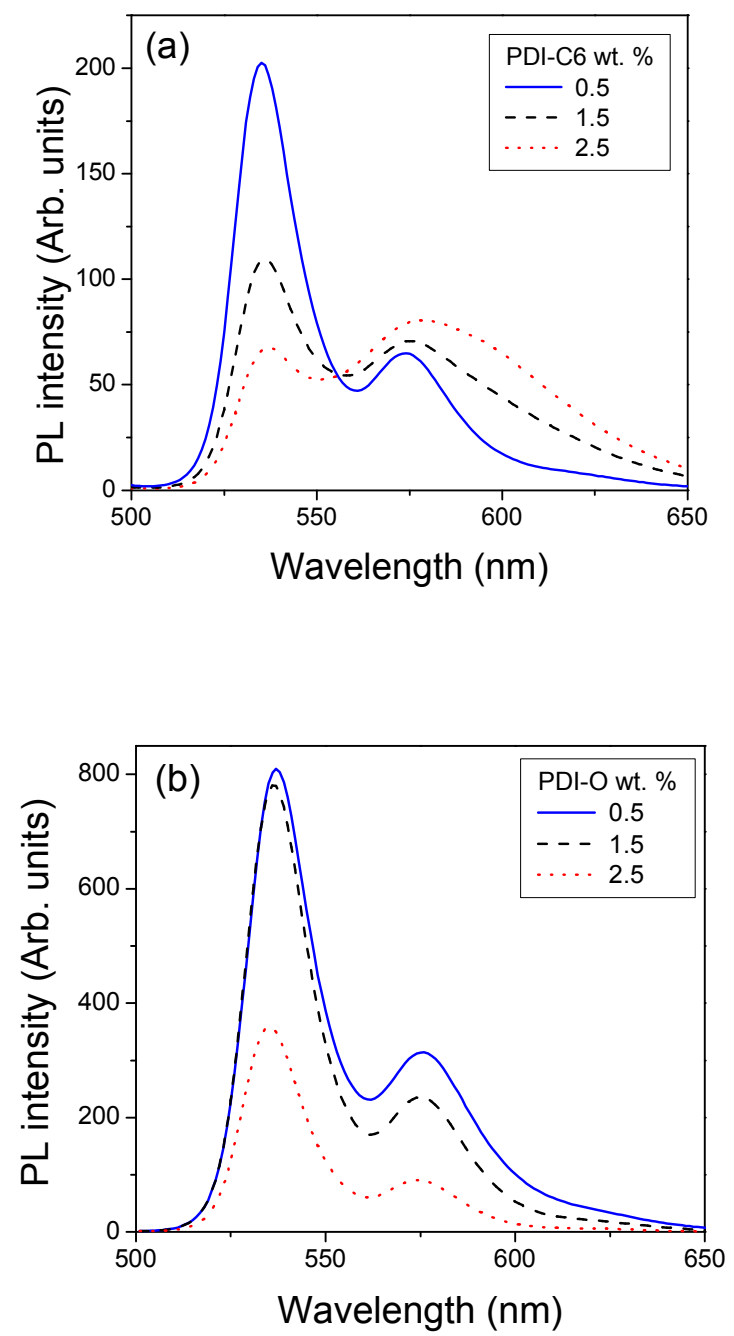

${ }_{5}$ Fig. 4. 


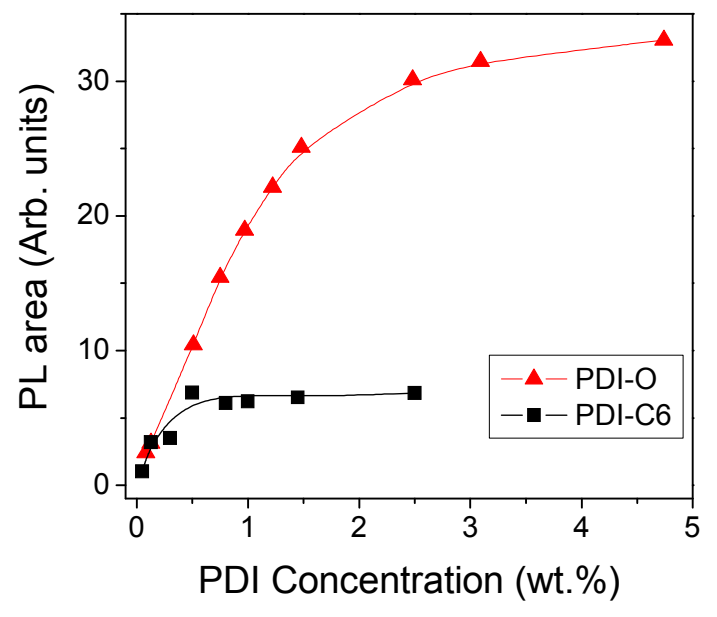

${ }_{5}$ Fig. 5. 


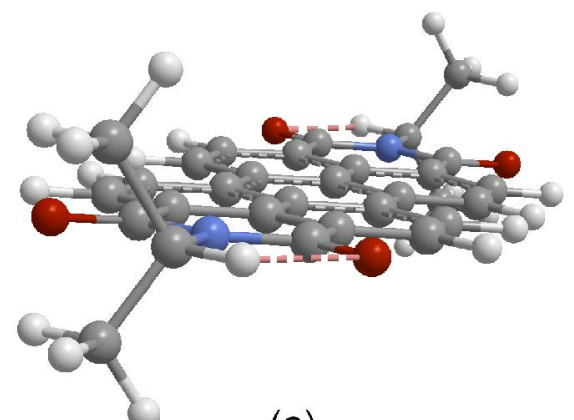

(a)

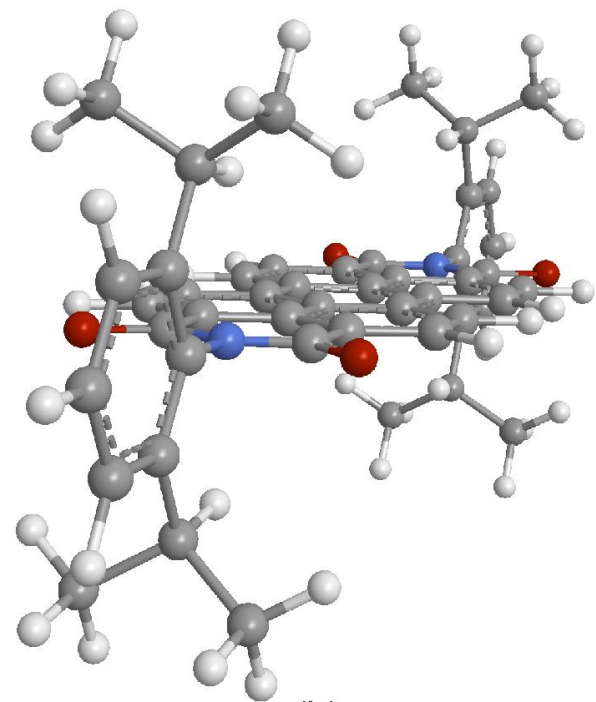

(b)

Fig. 6. 

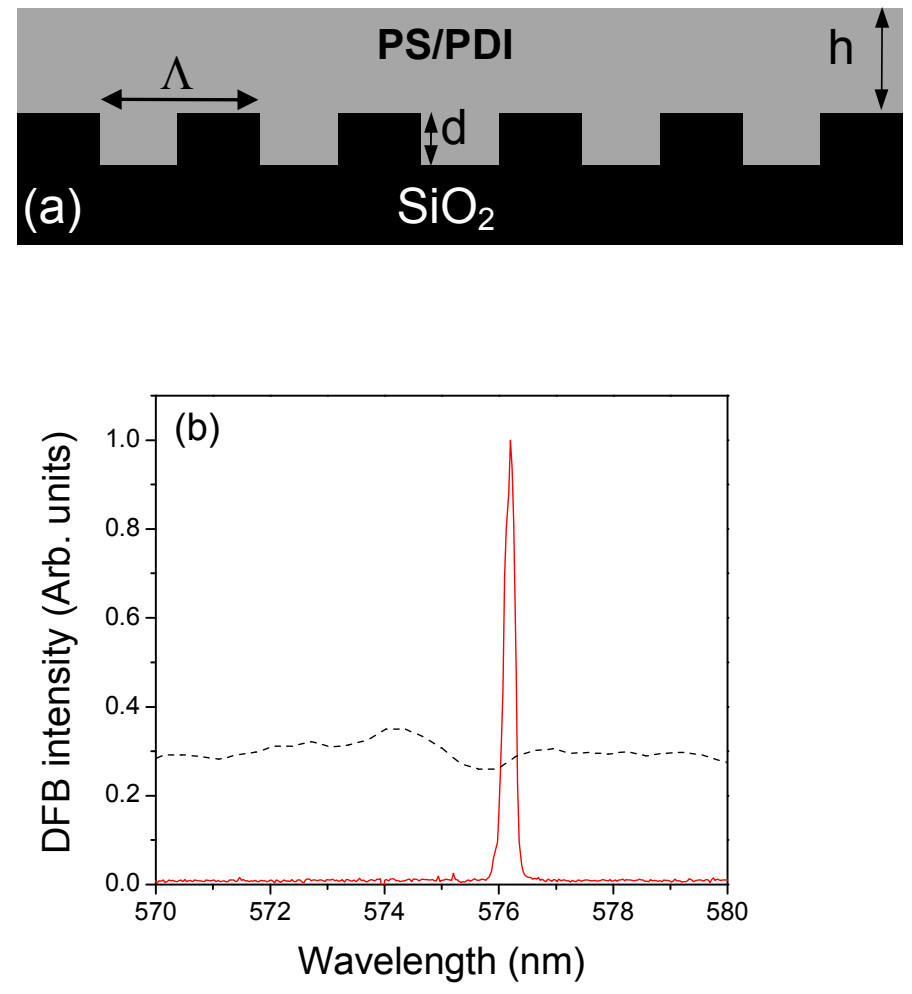

Fig. 7. 


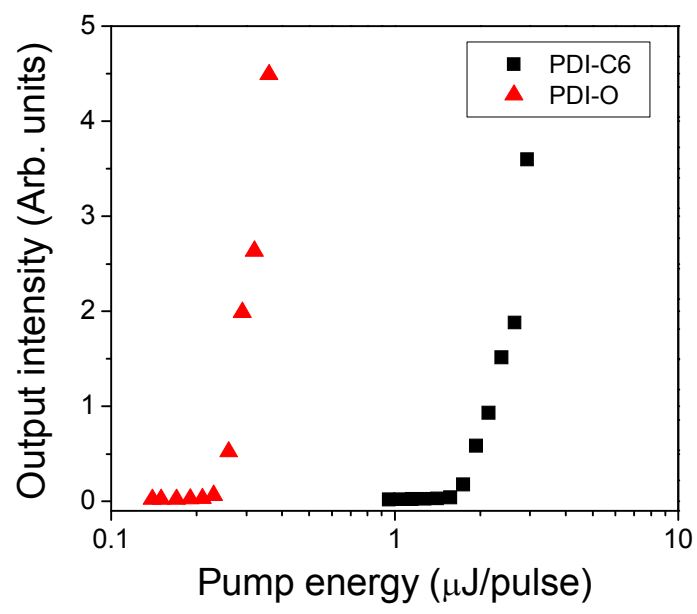

Fig. 8. 